\title{
Análise de protocolos com intervenção motora domiciliar para pacientes com doença de Alzheimer: uma revisão sistemática
}

\author{
Protocol analysis with home-based motor intervention for patients with Alzheimer's disease: a \\ systematic review
}

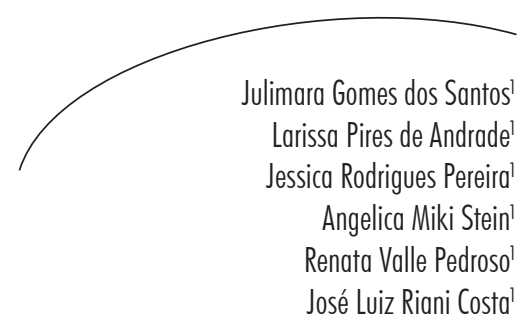

Resumo

A prática regular de atividade física é indicada como uma terapia não farmacológica ao tratamento da doença de Alzheimer por promover benefícios cognitivos, comportamentais e funcionais. Pouco se sabe, porém, sobre os protocolos com intervenção motora domiciliar para essa população. Pensando nisso, esta revisão teve como objetivo investigar e analisar os protocolos de intervenção motora domiciliar para idosos com doença de Alzheimer descritos em artigos científicos. Realizou-se busca sistemática, sem limite de data, nas seguintes bases de dados: Web of Science, PubMed, PsycINFO e Scopus. Utilizaram-se os seguintes operadores booleanos e palavras-chave: "homebased exercise" OR "home-based physical exercise" OR "home-based physical fitness" OR "home-based rehabilitation" OR "home-based physical therapy" OR "home-based physical activity" OR "home-based motor intervention" and "AD" OR "Alzheimer's disease" OR "Alzheimer" OR "Alzheimer's dementia". Realizou-se também uma busca manual nas listas de referência dos artigos selecionados. Dos cinco artigos que atenderam aos critérios de inclusão adotados, três realizaram um protocolo de intervenção motora domiciliar, conseguindo boa adesão ao programa, melhora geral da saúde e diminuição de sintomas depressivos. Os outros dois estudos limitaram-se a descrever os protocolos. Apesar de serem necessários mais estudos, com protocolos mais detalhados, esta revisão permitiu mostrar que protocolos de intervenção motora domiciliar também podem produzir efeitos positivos tanto para pacientes quanto para cuidadores.

\section{Abstract}

The regular practice of physical therapy is indicated as a non-pharmacological treatment of Alzheimer's disease by promoting cognitive, behavioral and functional benefits. However, little is known about the protocols with home-based motor intervention for this population. Thinking about it, this review aimed to investigate and analyze the protocols for home-based motor intervention for elderly with Alzheimer's disease described in scientific articles. A systematic search was performed in the following

Palavras-chave: Exercício Físico. Atividade Motora. Tratamento Domiciliar. Intervenção Domiciliar. Doença de Alzheimer. Revisão.

Departamento de Educação Física, Laboratório de Atividade Física e Envelhecimento, Instituto de Biociências. Universidade Estadual Paulista. Rio Claro, SP, Brasil. 
databases: Web of Science, PubMed, PsycINFO, and Scopus, using the following keywords and Boolean operators: "home-based exercise" OR "home-based physical exercise" OR "home-based physical fitness" OR "home-based rehabilitation" OR "homebased physical therapy" OR "home-based physical activity" OR "home-based engine Intervention" and "AD" OR "Alzheimer's disease" OR "Alzheimer " OR "Alzheimer's dementia". We also conducted a manual search of reference lists of selected articles. Of the five articles that met the inclusion criteria adopted, three performed a protocol for home motor intervention, achieving good compliance with the program, improvement of general health and reduction of depressive symptoms. The other two studies were limited to describing the protocols. Although more studies are needed, with detailed protocols, this review allowed to show that protocols for home motor intervention can also produce positive effects for both patients and caregivers.
Key words: Physical Exercise. Motor Activity. Residential Treatment. Home Intervention. Alzheimer Disease. Review.

\section{INTRODUÇÃO}

O aumento do envelhecimento populacional ocorrido nas últimas décadas tem provocado um crescimento na prevalência de doenças crônicas e neurodegenerativas, como é o caso da doença de Alzheimer (DA). Só no ano de 2010, estimouse que mais de 35 milhões de pessoas estavam vivendo com esse tipo de doença. ${ }^{1}$

Estudos post mortem indicam que a neuropatologia da DA envolve a presença de emaranhados neurofibrilares, formados pela hiperfosforilação da proteína tau, e placas senis, formadas pelo acúmulo de proteína beta-amiloide externas ao neurônio. Como consequência, ocorre a atrofia do hipocampo e, depois, de áreas corticais associativas, fazendo com que, no decorrer do seu quadro clínico, surjam alterações cognitivas e comportamentais. Nas fases mais avançadas da doença, ocorre também o declínio de capacidades funcionais, comprometendo a realização das atividades de vida diária e tornando o idoso dependente de cuidados. ${ }^{2-5}$

Para o tratamento, indica-se terapia farmacológica, mas também a não farmacológica, que pode incluir: estimulação cognitiva, convívio social $^{6,7}$ e prática regular de atividade física. ${ }^{8} \mathrm{~A}$ literatura científica tem mostrado que, de uma forma geral, a prática regular de atividade física é capaz de proporcionar benefícios cognitivos, comportamentais e funcionais, ${ }^{9-11}$ além de contribuir para um bom desempenho nas atividades de vida diária. ${ }^{12}$

Nota-se, no entanto, que a maioria das estratégias não farmacológicas, sobretudo a intervenção motora, em geral são desenvolvidas em clínicas ou universidades, o que pode dificultar o acesso ao tratamento, principalmente para aqueles que se encontram no estágio avançado da doença, por apresentarem maior comprometimento cognitivo e motor e, consequentemente, maior dificuldade de acesso a tratamentos fora de seu domićlio.

Nesses casos, uma alternativa que parece viável é a utilização de intervenções domiciliares. A literatura apresenta uma série de trabalhos que estudaram intervenções domiciliares ${ }^{13-17}$ com protocolos envolvendo, por exemplo, treinamento cognitivo e treinamento para cuidadores. Pouco se sabe, no entanto, sobre os protocolos que utilizaram intervenções motoras para esses pacientes, se esse tipo de estratégia não farmacológica é eficiente para o tratamento da doença de Alzheimer ou, ainda, que tipos de protocolo têm sido propostos e que resultados têm sido encontrados.

Este artigo teve como objetivo principal investigar e avaliar artigos científicos que descreveram e/ou utilizaram protocolos com intervenção motora domiciliar para pacientes com doença de Alzheimer. 


\section{MÉTODOS}

Esta revisão sistemática foi desenvolvida a partir da busca por artigos científicos, sem limite de data, em quatro bases de dados da área da saúde: Web of Science, PubMed, PsycINFO e Scopus. O período de busca foi de agosto a novembro de 2011, e foram utilizadas as seguintes palavraschave e operadores booleanos: "home-based exercise" OR "home-based physical exercise" OR "home-based physical fitness" OR "homebased rehabilitation" OR "home-based physical therapy" OR "home-based physical activity" OR "home-based motor intervention" and "AD" OR "Alzheimer's disease" OR "Alzheimer" OR "Alzheimer's dementia".

O processo de busca e seleção dos artigos ocorreu de forma sistemática, sendo realizado por um avaliador que selecionou primeiramente aqueles que apresentassem no título alguma das palavras-chave citadas. Depois foi feita a leitura dos resumos daqueles que passaram pela filtragem anterior. Por fim, foi realizada a leitura completa dos artigos que se enquadravam no tema proposto, para verificar se atendiam aos critérios de inclusão. Em um segundo momento, fez-se também uma busca manual nas listas de referência dos artigos selecionados.

Para que o artigo fosse incluído na revisão, deveria: (1) apresentar participantes com diagnóstico de doença de Alzheimer; (2) ter realizado ou descrito um protocolo com intervenção motora domiciliar como estratégia não farmacológica para pacientes com doença de Alzheimer; e (3) estar escrito nos idiomas inglês, espanhol ou português.
Respeitados os critérios de inclusão, foram excluídos da análise os artigos que: (1) não apresentavam um protocolo com intervenção motora domiciliar; (2) cuja amostra não era de pacientes com doença de Alzheimer; (3) a intervenção era voltada apenas para os cuidadores; e (4) apareceram duplicados em mais de uma base de dados.

A análise dos artigos baseou-se na descrição dos protocolos encontrados, explicitando seus pontos fortes e fracos, vislumbrando o desenvolvimento de estudos futuros.

\section{RESULTADOS}

A busca inicial com a utilização das palavraschave e operadores booleanos mencionados resultou em 60 artigos. Destes, 22 foram encontrados na PsycINFO, seis na Pubmed, 20 na Scopus e 12 na Web of Science.

Após a filtragem pela leitura dos títulos, 44 artigos foram excluídos por não se enquadrarem ao tema proposto, restando assim, 16 artigos. Em seguida, realizou-se a leitura dos resumos, e este número passou para dez artigos (incluindo os que apareceram em mais de uma base). Excluindo os que apareceram repetidamente nas bases de dados, restaram quatro artigos para serem lidos na íntegra. A partir da leitura, observou-se que todos esses artigos estavam de acordo com o objetivo deste estudo e atendiam aos critérios de inclusão. A busca manual na lista de referências bibliográficas dos artigos selecionados resultou em um artigo. A figura 1 apresenta de forma resumida a triagem dos artigos. 


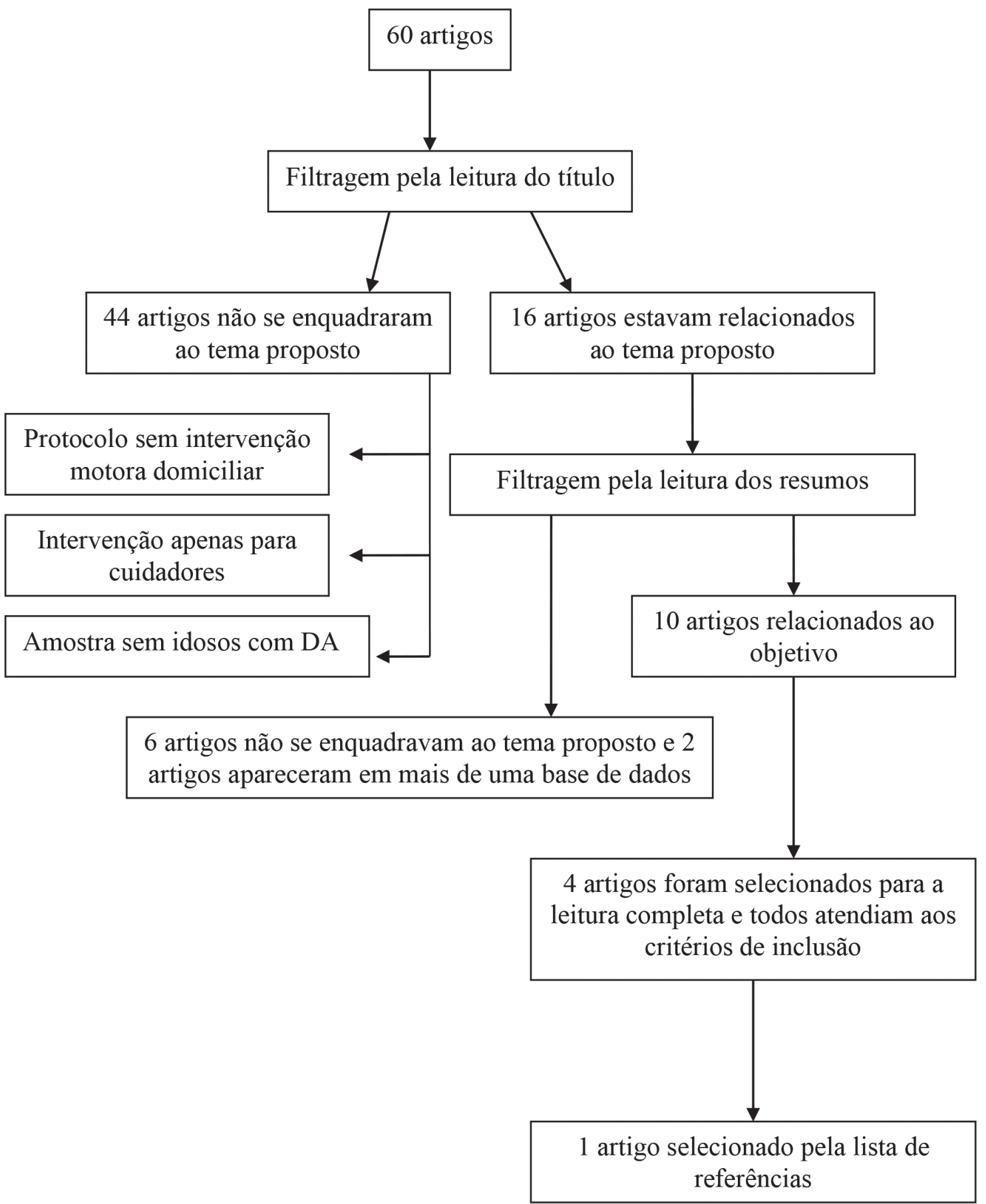

Figura 1 - Triagem dos artigos da revisão sistemática. 
Segue a descrição dos cinco estudos que foram analisados nesta revisão sistemática:

1) Teri et al. ${ }^{18}$ publicaram estudo realizado com 30 idosos com DA (78,7 $\pm 6,4$ anos) e seus

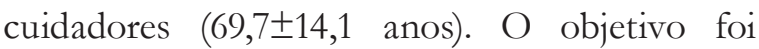
fornecer informações sobre a saúde física dessa população e estimulá-los à prática de exercício físico, e não avaliar a eficiência do programa sobre as capacidades físicas dos participantes. O programa abrangeu exercícios de equilíbrio, flexibilidade, força e resistência aeróbia. Para isso, os cuidadores receberam treinamento para que pudessem auxiliar os pacientes na execução dos exercícios. O protocolo de treinamento foi realizado na casa dos participantes, por profissionais da área da saúde (fisioterapeuta e assistente social). A duração do estudo foi de 12 semanas, sendo que nas primeiras três semanas, foram realizadas duas sessões semanais; nas quatro semanas seguintes, uma sessão semanal; e depois, quinzenais nas últimas quatro semanas. Os exercícios foram demonstrados pela primeira vez pelo treinador, depois o cuidador auxiliava o idoso a executar o exercício, sendo observado e assistido pelo treinador. Os componentes da capacidade funcional trabalhados durante as sessões, foram:

- Força: o treinamento de força ocorreu três vezes por semana em dias não consecutivos, intercalados com treinamento de resistência aeróbia. Os exercícios foram destinados aos membros inferiores (flexão plantar, dorsiflexão, flexão e extensão de joelho, abdução, extensão de quadril e marcha). Inicialmente, os exercícios foram realizados sem carga e depois, chegou-se à execução de duas séries de 12 repetições em cada exercício, com carga igual a cinco libras (aproximadamente 2,5 quilogramas).

- Equilíbrio e flexibilidade: foram indicados 10 a 15 minutos desse tipo de exercício e foram usados como aquecimento ou relaxamento para as atividades de fortalecimento ou de resistência aeróbia. O treinamento de equilíbrio contou com exercícios de transferência, exercícios de apoio e diferentes modos de andar (para trás, por exemplo). Os exercícios respeitaram uma progressão gradativa, partindo do mais fácil para o mais complexo. O treinamento de flexibilidade foi focado nas costas, ombros, quadris, isquiotibiais, gastrocnêmio, pescoço e mãos.

- Resistência aeróbia: foi indicado aos participantes que caminhassem ao menos 30 minutos em um mínimo de três dias não consecutivos por semana (alternados com dias de exercício de fortalecimento). Foi recomendado que começassem a andar no seu ritmo natural, aumentando gradualmente a velocidade da marcha. Aqueles que já estavam envolvidos em alguma forma alternativa de exercícios aeróbios regulares (por exemplo, dança de salão ou uso de bicicleta ergométrica) foram autorizados a substituir um ou mais dos dias de caminhada, por essa atividade alternativa.

Os exercícios recomendados foram descritos e ilustrados para facilitar o entendimento e execução dos mesmos. Além disso, os cuidadores receberam um diário onde deveriam registrar detalhadamente tudo o que ocorreu em cada sessão de intervenção (exercícios, quedas, ou qualquer informação sobre a saúde geral dos indivíduos). Os treinadores monitoraram semanalmente a adesão ao programa, por meio dos registros realizados nos diários.

Os pacientes foram avaliados quanto à velocidade de caminhada e equilíbrio. Além disso, o estado físico e funcional também foi avaliado utilizando-se duas escalas - Sickness Impact Profile (SIP) e Physical Functioning and Role Functioning (SF-36) - respondidas pelos cuidadores. Os cuidadores também foram questionados quanto aos dias em que seus familiares tiveram as atividades restritas e/ou ficaram de cama, nas últimas duas semanas, bem como quanto às situações de queda do último mês. A prática de exercício físico foi avaliada perguntando-se aos cuidadores o tempo gasto caminhando ou fazendo outra atividade aeróbia na última semana.

Os resultados mostraram que a amostra estudada apresentou um desempenho físico inferior quando comparado ao de idosos sem comprometimento cognitivo. Mas, de forma positiva, ao final do estudo foi alcançada uma 
boa adesão por parte dos participantes e os cuidadores mostraram-se capazes de aprender e auxiliar seus familiares com DA na execução de exercícios físicos.

2) Teri et al..$^{19}$ deram continuidade ao trabalho anterior, realizando um estudo controlado randomizado com 153 idosos com DA (78 \pm 6 anos) que abrangia um programa de exercícios domiciliares associado ao treinamento de cuidadores (70 \pm 13 anos) em técnicas de manejo comportamental. $\mathrm{O}$ estudo contou também com um grupo controle composto por idosos com DA ( $78 \pm 8$ anos) e seus cuidadores ( $70 \pm 13$ anos), mas que receberam apenas atendimento médico de rotina. Todos os participantes foram avaliados no momento da seleção da amostra (baseline), após três meses (período pós-tratamento) e aos seis, 12,18 e 24 meses (follow-up) por meio de uma avaliação "cega". O programa de exercícios domiciliares incluía exercícios de resistência aeróbia, de força, equilíbrio e flexibilidade. Foram realizadas 12 sessões supervisionadas, com duração de uma hora cada. Nas primeiras três semanas, a frequência foi de duas sessões por semana. Nas quatro semanas seguintes, realizouse uma sessão por semana; e durante as quatro últimas semanas, a frequência foi quinzenal. $\mathrm{O}$ objetivo era que os participantes realizassem ao menos 30 minutos diários de exercícios.

O treinamento de gestão comportamental envolveu instruções aos cuidadores sobre como identificar e modificar os distúrbios de comportamento apresentados pelos pacientes, assim como orientação sobre a própria doença. Além disso, foi sugerido que estimulassem os pacientes a aumentar sua atividade física e social. Após o tratamento, os participantes do grupo intervenção apresentaram melhora geral da saúde, avaliada pelo SF-36 e SIP mobilidade (mobility), bem como diminuição dos sintomas depressivos avaliados pela escala de Cornell, enquanto o grupo controle piorou. Observou-se que os participantes do grupo intervenção realizaram atividades físicas extras àquelas recomendadas pelo protocolo, havendo, portanto, aumento do nível de atividade física semanal desse grupo. Além disso, parece que o programa contribuiu para retardar a institucionalização dos pacientes, geralmente causada pela dificuldade dos cuidadores em gerir os distúrbios comportamentais.

Terminado o estudo, sessões extras de intervenção foram realizadas visando ajudar os cuidadores e os pacientes a manterem o que foi ensinado. Dos 153 idosos que começaram o estudo, 89 concluíram as avaliações do follow-up, cujos resultados mostraram que as diferenças significativas entre o grupo intervenção e o grupo controle foram novamente obtidas sobre a subescala de funcionamento físico SF-36 e a escala SIP mobilidade (mobility). A principal causa que levou os pacientes a não cumprirem todas as avaliações foi a institucionalização, não havendo diferenças significativas entre os grupos (67\% do grupo controle e $68 \%$ do grupo intervenção; $\mathrm{p}=0,84)$. Apesar disso, os autores notaram que um maior número de participantes do grupo controle foi institucionalizado devido à presença de distúrbios comportamentais, quando comparado ao grupo que recebeu intervenção domiciliar.

3) $\mathrm{O}$ estudo de Steinberg et al. ${ }^{20}$ avaliou um programa de exercício domiciliar com características de um estudo piloto. Participaram 27 idosos com DA divididos em grupo intervenção (76,5 \pm 3 ,9anos) e grupo controle (74,0 \pm 8 ,1anos). O programa de exercícios foi desenvolvido por um profissional da área da Fisiologia do Exercício, que instruiu os participantes para a prática diária de exercícios que trabalhavam quatro componentes: aeróbio, força, flexibilidade e equilibrio. Quando realizavam a tarefa completa, os participantes ganhavam dois pontos, e quanto faziam apenas uma parte, computavam um ponto. O objetivo era somar seis pontos de atividade aeróbia por semana e quatro pontos nos componentes restantes. O grupo controle recebeu visitas para avaliação da segurança em casa e a partir disso foram feitas algumas recomendações de adaptação domiciliar. 
Quanto à atividade física, foi solicitado aos participantes deste grupo que registrassem três atividades físicas que realizassem regularmente. Cuidadores de ambos os grupos receberam diários para que descrevessem as tarefas concluídas semanalmente. Os resultados mostraram que o programa teve boa adesão. Pacientes do grupo exercício apresentaram tendência para melhora nos testes manuais e de força de membros inferiores. Esse grupo, no entanto, apresentou pior desempenho nas avaliações de depressão e qualidade de vida.

4) Cyarto et al. ${ }^{21}$ descreveram o protocolo de um estudo futuro, que será controlado e randomizado. Para este estudo, pretendemse recrutar 230 idosos com DA que serão divididos em:

- Grupo de intervenção domiciliar: abrangerá programa de atividade física (AF), intervenção comportamental e monitoramento por telefone, com duração total de 24 semanas. O programa de atividade física compreenderá 150 minutos de AF moderada por semana. Para esse grupo, será realizado também um workshop de atividade física, envolvendo cuidadores e pacientes, onde será entregue um manual do programa contendo instruções sobre os exercícios e como realizá-los com segurança, além de um diário para que registrem as sessões. O programa será individualizado, baseado nos interesses pessoais dos participantes, bem como suas limitações de saúde. Assim, os pacientes serão instruídos a progredirem gradualmente até que atinjam a meta de AF em oito semanas. Aqueles que já realizarem 150 minutos de AF por semana serão incentivados a adicionar uma sessão de 50 minutos. Os cuidadores serão convidados a realizar os exercícios junto aos participantes e, a fim de reduzir a sobrecarga sobre os mesmos, será permitido aos participantes realizar as atividades em centros comunitários.

Após as 24 semanas de intervenção, os participantes serão convidados a continuar com o programa de AF por mais 24 semanas, mas sem acompanhamento ou contato com os pesquisadores, exceto para as avaliações subsequentes. Assim, ao final dos 12 meses, eles responderão um questionário quanto à sua adesão ao programa anterior, mantendo as atividades desenvolvidas nos primeiros seis meses. A intervenção comportamental será baseada nas etapas de um modelo de mudança de comportamento para adoção da prática de AF. Serão oferecidas oficinas que abordem temas como os benefícios e as recompensas do exercício físico, a fixação de metas, a gestão do tempo, entre outros, buscando motivar os participantes e aumentar a adesão ao programa.

Regularmente, durante as 24 semanas do programa, serão enviados boletins com informações motivacionais, além de telefonemas para monitorar e dar feedback sobre o progresso de cada um e incentivar a adesão contínua. Ao final do estudo, todos os participantes receberão um relatório de suas avaliações.

- Grupo de cuidados habituais: receberá material educativo sobre a DA e recomendações sobre um estilo de vida saudável. Também serão contatados por telefone para garantir que ambos os grupos recebam tratamento semelhante. Ao final do estudo, esses participantes terão o direito de acompanhar uma sessão de atividade física.

5) Pitkala et al. ${ }^{22}$ descreveram o protocolo de um estudo de intervenção que será controlado e randomizado, com duração total de um ano ou até que o paciente precise ser internado para cuidado institucional permanente. A amostra será composta por 210 pacientes com DA e dividida em três grupos, sendo dois de intervenção ativa (um deles com tratamento de reabilitação realizado em casa e outro em um centro-dia) e um terceiro grupo que manterá o tratamento usual - grupo controle). As avaliações ocorrerão no início, e aos três, seis e 12 meses. Um geriatra será responsável pela avaliação e montagem do programa de reabilitação, incluindo também a parte nutricional. $O$ grupo de intervenção domiciliar receberá duas visitas por semana de 
uma hora de duração, quando serão orientados exercícios de acordo com as necessidades do paciente e do cuidador, incluindo, por exemplo, orientações sobre como realizar transferências, treinamento de equilíbrio, treinamento para funções executivas e tarefa dupla.

O grupo do centro-dia receberá um treinamento físico diversificado de quatro horas/dia e duas vezes por semana, com exercícios de resistência, equilíbrio, força, tarefa dupla e exercícios direcionados para melhorar o funcionamento das funções executivas. Ambos os grupos de intervenção terão orientação de fisioterapeutas para realização das atividades. Os pacientes do grupo controle não receberão intervenção, mas manterão os cuidados habituais oferecidos pelo sistema de saúde e receberão também aconselhamento sobre nutrição e exercício.

As características gerais e resultados dos estudos estão apresentados no quadro 1 . 


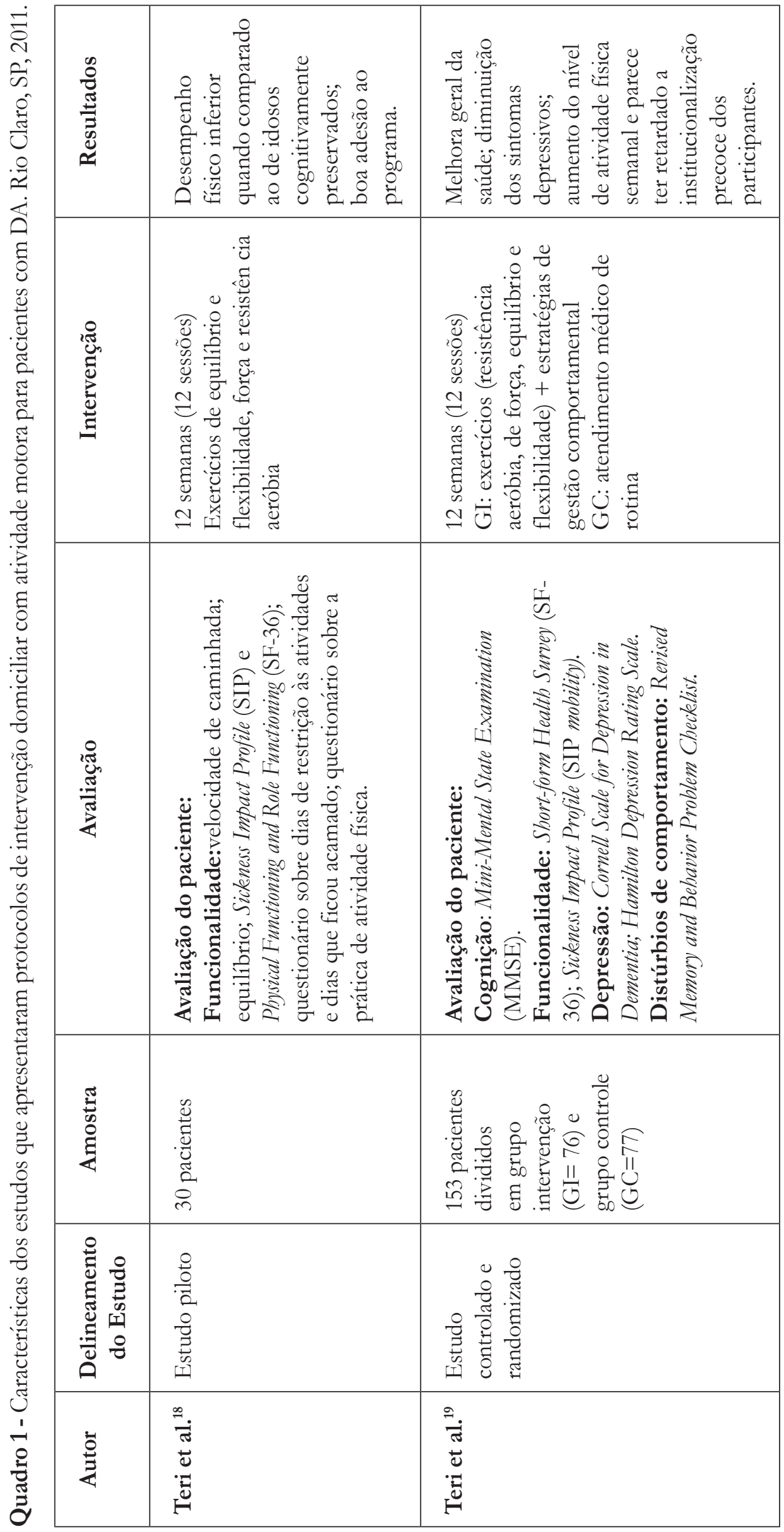




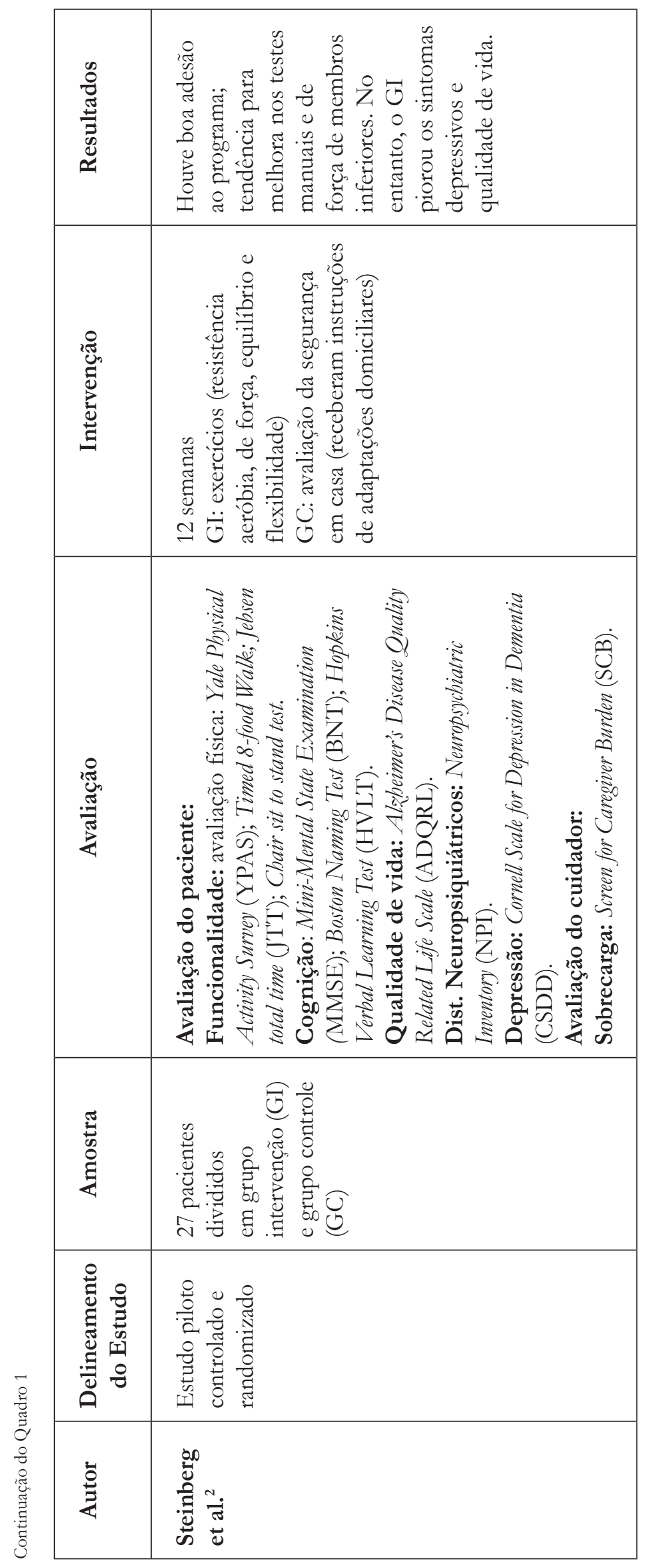




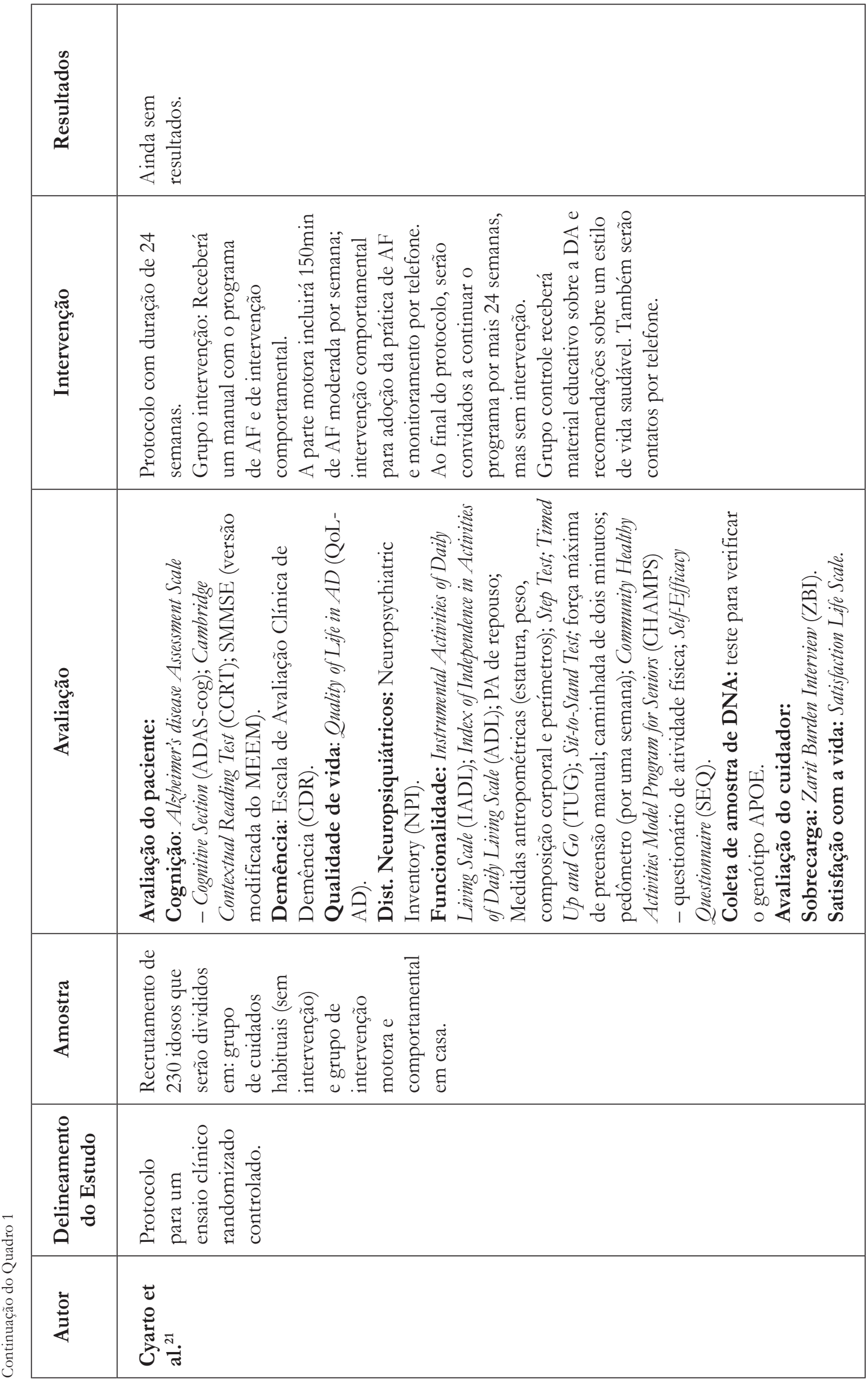




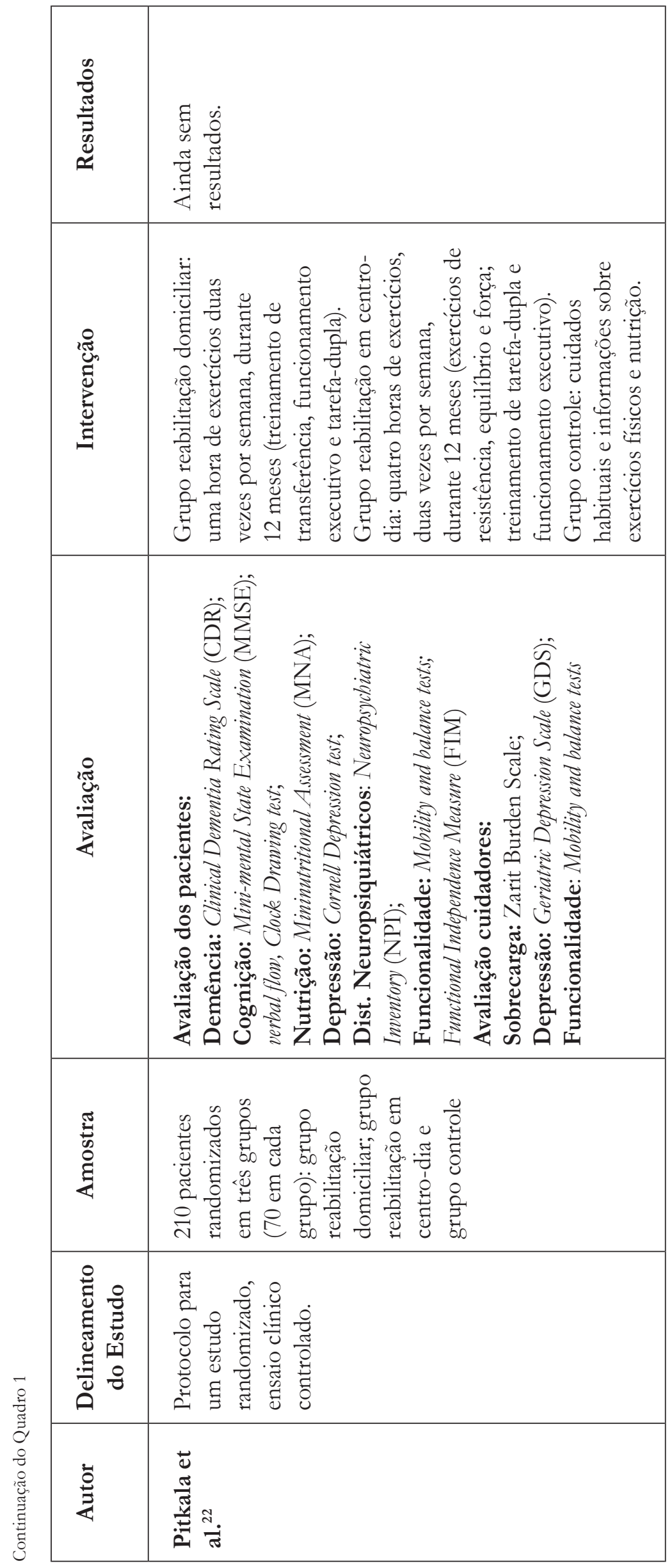




\section{DISCUSSÃO}

Como se observa, os poucos estudos encontrados foram publicados recentemente, demonstrando a escassez de estudos nessa linha, bem como a dificuldade em colocar em prática protocolos que contam com tamanha especificidade. Estes demandam intensa colaboração dos cuidadores familiares $\mathrm{e}$ profissionais, além de um controle rigoroso para que as atividades sejam feitas como planejado e de maneira segura, principalmente quando não há presença de um profissional em todas as sessões.

Assim, dos cinco estudos incluídos na análise desta revisão, verificou-se que apenas dois (estudos 2 e 3) apresentavam os resultados do efeito da intervenção motora. O estudo 1 foi caracteristicamente um estudo piloto que não teve como objetivo principal avaliar o efeito da intervenção sobre as variáveis motoras, e os estudos 4 e 5 ficaram restritos à descrição de protocolos, o que de certa forma dificulta tecer maiores críticas sobre a eficácia dos métodos.

No entanto, como o objetivo central desta revisão sistemática foi investigar e analisar os protocolos de intervenção motora domiciliar descritos na literatura, foi dada atenção ao caráter metodológico de cada um deles, enfatizando-se os protocolos descritos.

Para facilitar a análise, os artigos foram expostos seguindo uma linha cronológica, onde se discutiu primeiro o que já foi feito em termos de protocolo de intervenção e, depois, as propostas feitas pelos estudos mais recentes. Com base nisso, buscou-se chegar o mais próximo possível ao protocolo de intervenção motora domiciliar mais adequado para idosos com doença de Alzheimer e quais caminhos os estudos futuros deverão seguir.

O trabalho de Teri et al., ${ }^{18}$ apesar de não ter como foco avaliar o efeito da intervenção motora domiciliar sobre os pacientes com DA, pode ser considerado um trabalho inovador e de extrema importância, já que de acordo com esta revisão, foi o primeiro a abordar essa temática e acabou servindo de base para estudos posteriores. Com ele foi possível atestar a viabilidade de protocolos desse tipo.

Os estudos de Teri et al. ${ }^{19}$ (estudo 2) e Steinberg et al. ${ }^{20}$ (estudo 3) apresentaram algumas semelhanças, como: avaliação primária da funcionalidade motora, presença de grupo controle, randomização da amostra, avaliadores cegos quanto ao tratamento e um período de intervenção de 12 semanas.

Apesar de o estudo 2 ter contado com maior número de participantes que o do estudo 3 , este, por sua vez, realizou um protocolo de avaliação mais completo, incluindo avaliação da cognição, dos sintomas neuropsiquiátricos e da qualidade de vida. Além disso, avaliaram também o efeito da intervenção sobre a sobrecarga do cuidador.

Com relação aos testes escolhidos, verificouse que eram destinados à população idosa, mas poucos eram específicos para idosos com DA, o que reforça a dificuldade de se encontrar instrumentos validados para esse público na literatura atual.

Quanto ao protocolo de intervenção, ambos os programas indicaram exercícios diários que trabalhassem os mesmos componentes: resistência aeróbia, força, flexibilidade e equilíbrio, assim como recomenda o Colégio Americano de Medicina e Esporte para idosos (ACSM) ${ }^{23}$ No entanto, apenas os estudos $1 \mathrm{e}$ 2 apresentaram os protocolos de maneira mais detalhada; os outros poderiam ter sido mais claros quanto à descrição dos materiais utilizados, bem como os exercícios realizados e as repetições adotadas. Isso facilitaria a replicação de um protocolo inovador como esse.

Ainda sobre a intervenção, o estudo 2 mostrou-se diversificado, abrangendo tanto o aspecto motor quanto o comportamental, enquanto o estudo 3 foi puramente motor. Os participantes do estudo 2 tinham como meta realizar ao menos 30 minutos de exercícios diários. O estudo 3, por sua vez, apresentou de maneira hierárquica como deveria ser a realização dos exercícios, priorizando os aeróbios. 
O estudo 3, como não contou com a supervisão de um profissional em todas as sessões domiciliares, buscou uma alternativa para controlar a realização das tarefas, entregando uma espécie de diário aos cuidadores para que anotassem as atividades concluídas durante a semana.

Comparando-se os grupos controle, percebese que apenas o grupo do estudo 2 não recebeu intervenção alguma. Já no estudo 3, apesar de não ter havido orientação quanto à prática de atividade física, de certa forma tratou de outro grupo de intervenção, já que estes receberam visitas onde foram dadas instruções sobre adaptação do domicílio. Dessa forma, os grupos desse estudo diferenciaram-se apenas quanto à realização ou não dos exercícios.

De forma geral, ambos os estudos apresentaram resultados positivos quanto à estratégia de intervenção, demonstrando a viabilidade da realização de estudos como estes e a eficácia dos mesmos, melhorando o desempenho funcional dos participantes. No entanto, só o programa do estudo 2 foi capaz de reduzir sintomas depressivos, dando indícios de que protocolos diversificados talvez sejam os mais indicados para o tratamento tanto de aspectos físicos quanto psíquicos desses pacientes. Os autores do estudo 3, no qual se observou "piora" nos sintomas depressivos e na qualidade de vida, justificam esses resultados pela natureza piloto do estudo; o pequeno tamanho da amostra, que provavelmente limitou a inferência estatística, ou ainda, a possibilidade de terem sido descobertas casuais. Tais resultados, no entanto, podem estar relacionados ao próprio processo progressivo da doença de Alzheimer.

Mais tarde, em 2010, outros dois estudos foram publicados descrevendo protocolos de intervenções semelhantes: Cyarto et al. ${ }^{21}$ (estudo 4) e Pitkala et al. ${ }^{22}$ (estudo 5). Já de antemão, esses dois protocolos se diferenciam dos demais na intenção de recrutar um grande número de participantes, mais de $200 \mathrm{em}$ cada um. Para isso, os autores usarão como estratégia a descentralização do estudo, contando com o apoio de outros centros de pesquisa.
O tempo de intervenção também será maior nos estudos 4 e 5: 12 meses no estudo 4 e 24 semanas no estudo 5, com possibilidade de se estender por mais 24 semanas, porém sem presença da equipe de pesquisa. Cabe ressaltar que longos períodos de pesquisa demandam rigoroso controle por parte dos pesquisadores para que a perda amostral não seja grande.

Ambos os estudos procurarão randomizar a amostra e utilizar grupo controle para comparação. Porém, o estudo 5 incluirá um segundo grupo de intervenção, a ser desenvolvido em um centro-dia. Dessa forma, mais do que comparar intervenções realizadas em locais diferentes, eles irão aplicar protocolos diferentes quanto aos exercícios e duração das sessões.

No estudo 5, durante a descrição do protocolo do grupo que receberá intervenção domiciliar, os autores não incluíram - ao menos não de forma explícita - o treinamento de componentes da capacidade funcional (resistência aeróbia, equilíbrio, força e flexibilidade), ficando o grupo do centro-dia com uma intervenção mais abrangente. No entanto, não há no texto uma justificativa para adoção de tais procedimentos.

Sobre os grupos controle, nota-se que, de certa forma, ambos receberão intervenção, aqui tratada como educacional, já que terão acesso a informações sobre exercícios físicos e nutrição (estudo 5) ou material educativo sobre a DA, recomendações sobre um estilo de vida saudável, além de contato por telefone (estudo 4). Ao mesmo tempo em que essa abordagem se mostra importante e necessária, pode-se questionar o porquê de o estudo 5 não pretender levar esse tipo de informação também ao grupo de intervenção domiciliar, tendo em vista a grande dificuldade que os cuidadores enfrentam para cuidar de seus familiares, na maioria das vezes sem possuir instrução suficiente e apoio adequado.

Já o estudo 4 terá um delineamento mais simplificado, contando com apenas dois grupos, no qual o grupo intervenção deverá realizar 150 minutos de AF moderada por semana, o que é recomendado para pessoas idosas. No entanto, 
os autores não relatam quais exercícios serão prescritos, nem quais componentes da capacidade física serão treinados; mencionam apenas que a prescrição levará em conta a capacidade física e os interesses dos participantes. Eles justificam a utilização dessa estratégia como alternativa para manter a adesão e o interesse dos participantes no programa.

Alguns outros pontos interessantes do estudo 4 merecem destaque:

- Todo o programa estará descrito sob a forma de um manual, que será entregue aos cuidadores, facilitando a execução das atividades.

- Os cuidadores também serão incentivados a realizar as atividades junto com os pacientes. Como em sua grande maioria os cuidadores são também idosos, estratégias para adoção de uma vida mais saudável se torna muito importante, devido ao desgaste físico e mental causado pela função de cuidar de alguém dependente, que pode fazer dependente também o cuidador. ${ }^{24}$

- Haverá um monitoramento por telefone. Além de contribuir para o controle da execução das atividades e servir como meio motivacional dos participantes, essa abordagem aproxima o pesquisador do sujeito da pesquisa, podendo servir ainda como um momento para que as dúvidas sejam sanadas e o protocolo seja seguido com mais segurança.

Com relação aos instrumentos de avaliação utilizados nos dois estudos, observa-se que ambos tiveram o cuidado de incluir testes também para os cuidadores, indo o estudo 5 além, ao propor avaliação da funcionalidade desses cuidadores. Como já mencionado, cuidadores idosos necessitam de atenção ainda maior, por apresentarem os efeitos deletérios do envelhecimento potencializado pelo desempenho dessa árdua função. Uma avaliação da capacidade funcional de cuidadores pode ainda ser utilizada como critério de inclusão em estudos futuros, já que protocolos como esses necessitam da participação ativa dos cuidadores, devendo estes apresentar condições físicas de auxiliar os pacientes na execução dos exercícios.

Sobre a bateria de avaliação destinada aos pacientes, o estudo 4 apresentou uma diversidade de testes que vão desde testes cognitivos, de qualidade de vida, distúrbios neuropsiquiátricos, funcionalidade motora, até teste para verificação da expressão gênica da apoE para analisar uma possível relação entre esse genótipo e os benefícios da atividade física. Apesar de outros genes estarem relacionados com a doença de Alzheimer, a literatura tem demonstrado que $\mathrm{O}$ alelo $\varepsilon 4$ do gene apoE é tido como o de maior suscetibilidade para o surgimento da doença. ${ }^{25,26}$ Isso demonstra a dimensão do estudo e o quanto os autores estão atualizados quanto às recentes linhas de investigação sobre a doença de Alzheimer. Resta, porém, esperar novas publicações desses autores com a divulgação dos resultados de protocolos tão inovadores.

Embora esta revisão apresente uma importante limitação quanto ao reduzido número de estudos, em contrapartida traz à tona uma discussão atual e relevante, que poderá servir de incentivo para a produção de novos estudos na área.

\section{CONCLUSÃO}

Com base nesta revisão, foi possível perceber que estudos com intervenção motora domiciliar para idosos com doença de Alzheimer são recentes e escassos, evidenciando a necessidade de mais investigação sobre o tema. Apesar disso, os estudos aqui apresentados evidenciam que esse tipo de tratamento não farmacológico é benéfico, tanto para pacientes quanto para seus cuidadores, e que estes, se receberem treinamento e orientação adequados, são capazes de auxiliar no tratamento de seus familiares, estimulando e ajudando na execução das atividades prescritas pelo profissional da saúde. 
Sugere-se que os estudos posteriores atentem para a resposta a algumas questões, a fim de preencherem as lacunas ainda existentes, como por exemplo:

- Será que os benefícios relatados nos estudos seriam alcançados em pacientes mais debilitados, que estivessem no estágio avançado da doença? Para isso, é preciso que os próximos estudos apresentem as amostras separadas, de acordo com o estágio da doença.

- A inclusão de estimulação cognitiva no protocolo traria benefícios mais evidentes nessas funções?

- A inclusão de um treinamento específico das atividades da vida diária, como destinar um tempo da intervenção para auxiliar/

\section{REFERÊNCIAS}

1. Alzheimer's Disease International. Relatório sobre a Doença de Alzheimer no mundo de 2009. Resumo Executivo. Londres: Alzheimer's Disease International; 2010.

2. Caramelli P, Barbosa MT. Como diagnosticar as quatro causas mais freqüentes de demência? Rev Bras Psiquiatr 2002;24(Supl I):7-10.

3. Bertolucci PHF. Manual do Cuidador Doença de Alzheimer nas Fases Moderada e Grave. São Paulo: EPM - UNIFESP; 2007.

4. Melo MA, Driusso P. Proposta Fisioterapêutica para os cuidados de Portadores da Doença de Alzheimer. Envelhecimento e Saúde 2006;12(4):11-8.

5. Paula JA, Roque FP, Araujo FS. Qualidade de vida em cuidadores de idosos portadores de demência de Alzheimer. J Bras Psiquiatr 2008;57(4):283-7.

6. Heyn P, Abreu BC, Ottenbacher KJ. The effects of exercise training on elderly persons with cognitive impairment and dementia: a meta-analysis. Arch Phys Med Rehabil 2004;85(10):1694-704.

7. Arcoverde C, Deslandes A, Rangel A, Pavão R, Nigri F, Engelhardt E, et al. Role of physical activity on the maintenance of cognition and activities of daily living in elderly with Alzheimer's disease. Arq Neuropsiquiatr 2008;66(2B):323-7. estimular o paciente a pentear os cabelos, vestir-se ou escovar os dentes, afetaria de forma positiva e direta o desempenho para essas atividades?

- Mesmo que, às vezes, os testes não consigam mostrar objetivamente a melhora de determinada função, será que de maneira qualitativa esses resultados não poderiam ser vistos?

\section{AGRADECIMENTOS}

À Coordenação de Aperfeiçoamento de Pessoal de Nível Superior (CAPES), à Fundação de Amparo à Pesquisa do Estado de São Paulo - FAPESP ( $n^{\circ}$ do processo 2012/138137) e ao Laboratório de Atividade Física e Envelhecimento (LAFE).
8. Coelho FGM, Santos-Galduroz RF, Gobbi S, Stella F. Atividade física sistematizada e desempenho cognitivo em idosos com demência de Alzheimer: uma revisão sistemática. Rev Bras Psiquiatr 2009;31(2):163-70.

9. Coelho FGM, Andrade LP, Pedroso RV, SantosGalduroz RF, Gobbi S, Costa JL, et al. Multimodal exercise intervention improves frontal cognitive functions and gait in Alzheimer's disease: a controlled trial. Geriatr Gerontol Int 2013;13(1):198-203.

10. Pedroso RV, Coelho FGM, Santos-Galduroz RF, Costa JL, Gobbi S, Stella F. Balance, executive functions and falls in elderly with Alzheimer's dementia (AD): a longitudinal study. Arch Gerontol Geriatr 2012;54(2):348-51.

11. Canonici AP, Andrade LP. Gobbi S, Santos-Galduroz RF, Gobbi LT, Stella F. Functional dependence and caregiver burden in Alzheimer's disease: a controlled trial on the benefits of motor intervention. Psychogeriatrics 2012;12(3):186-92.

12. Garuffi M, Costa JL, Hernández SS, Vital TM, Stein AM, dos Santos JG, et al. Effects of resistance training on the performance of activities of daily living in patients with Alzheimer's disease. Geriatr Gerontol Int 2013;13(2):322-8. 
13. Vicente MM, Delgado MG, Fuertes NG, Prieto JP. Efectos de un programa de ejercicio físico a domicilio en cuidadores de pacientes de Alzheimer: un estudio piloto. Revista de Psicologia del Deporte 2009;18(2):255-70.

14. Vidovich MR, Shaw J, Flicker L, Almeida OP. Cognitive activity for the treatment of older adults with mild Alzheimer's disease (AD) - PACE AD: study protocol for a randomised controlled trial. Trials 2011;12:47.

15. Farran CJ, Staffileno BA, Gilley DW, McCann JJ, Yan Li, Castro CM, et al. A lifestyle physical activity intervention for caregivers of persons with Alzheimer's disease. Am J Alzheimers Dis Other Dem 2008;23(2):132-42.

16. Hanser SB, Butterfield-Whitcomb J, Kawata M, Collins BE. Home-based music strategies with individuals who have dementia and their family caregivers. J Music Ther 2011;48(1):2-27.

17. Pitrou I. Well-being of patients with dementia and their caregivers after a biobehavioral home-based intervention. JAMA 2010;304(22):2482-3.

18. Teri L, McCurry SM, Buchner DM, Logsdon RG, LaCroix AZ, Kukull WA, et al. Exercise and activity level in Alzheimer's disease: a potential treatment focus. J Rehabil Res Dev 1998;35(4):411-9.

19. Teri L, Gibbons LE, McCurry SM, Logsdon RG, Buchner DM, Barlow WE, et al. Exercise plus behavioral management in patients with Alzheimer disease: a randomized controlled trial. JAMA 2003;290(15):2015-22.
20. Steinberg M, Leoutsakos JM, Podewils LJ, Lyketsos CG. Evaluation of a home-based exercise program in the treatment of Alzheimer's disease: the Maximizing Independence in Dementia (MIND) study. Int J Geriatr Psychiatry 2009;24(7):680-5.

21. Cyarto EV, Cox KL, Almeida OP, Flicker L, Ames D, Byrne G, et al. The fitness for the Ageing Brain Study II (FABS II): protocol for a randomized controlled clinical trial evaluating the effect of physical activity on cognitive function in patients with Alzheimer's disease. Trials 2010;11:120.

22. Pitkala KH, Raivio MM, Laakkonen ML, Tilvis RS, Kautiainen H, Strandberg TE. Exercise rehabilitation on home-dwelling patients with Alzheimer's disease a randomized, controlled trial. Study protocol. Trials 2010;11:92

23. American College of Sports Medicine, Chodzko-Zaiko WJ, Proctor DN, Fiatarone Singh MA, Minson CT, Nigg CR, et al. American College of Sports Medicine position stand. Exercise and physical activity for older adults. Med Sci Sports Exerc 2009;41(7):1510-30.

24. Cruz MN, Hamdan AC. O impacto da doença de Alzheimer no cuidador. Psicologia em Estudo 2008;13(2):223-9.

25. Laws SM, Hone E, Gandy S, Martins RN. Expanding the association between the APOE gene and the risk of Alzheimer's disease: possible roles for APOE promoter polymorphisms and alterations in APOE transcription. J Neurochem 2003;84(6):1215-36.

26. Bertram L, McQueen MB, Mullin K, Blacker D, Tanzi RE. Systematic meta-analyses of Alzheimer disease genetic association studies: the AlzGene database. Nat Genet 2007;39(1):17-23. 